\title{
Severe Thrombocytopenia Cured After Ovarian Tumours Surgical Removal
}

\section{Cosmina Andreea Catoiu ${ }^{1}$, Ioan Corneliu Tanase ${ }^{1,2 *}$, Bogdan Gabriel Stoica $^{1,2}$ and Sorin Constantin Paun ${ }^{1,2}$}

${ }^{1}$ Department of Surgery, Emergency Hospital - Bucharest, Romania

${ }^{2}$ University of Medicine and Pharmacy Carol Davila Bucharest, Romania

*Corresponding Author: Ioan Corneliu Tanase, University of Medicine and Pharmacy Carol Davila Bucharest, Department of Surgery, Emergency

Hospital - Bucharest, Romania.
Received: October 27, 2021

Published: November 10, 2021

(C) All rights are reserved by Ioan Corneliu

Tanase., et al.

\begin{abstract}
Autoimmune thrombocytopenia (ITP) is an acquired immune-mediated disorder characterized by early platelet destruction due to antiplatelet autoantibodies. Although progress in the immunopathology of ITP has been done, pathogenesis of ITP remains yet to be determined, and currently there are no specific tests that can diagnose this disease, often being a diagnosis of exclusion. We describe the case of a 65-year-old woman admitted in the surgical department with progressively worsening abdominal distension for the past 12 months. Imagistic investigations showed a giant cystic tumour with a distinct septum and inhomogeneous internal content, which extended from the pelvis all the way through the upper abdomen, suggesting a complex left ovarian cyst with no lymph nodes or other intraabdominal tumoural masses. Blood tests showed mild anaemia, and severe thrombocytopenia with a platelet count of $21.000 / \mu \mathrm{L}$ refractory to treatment, anisocytosis with micro- and macro-thrombocytes. Coagulation profile was normal. The patient underwent, total hysterectomy with bilateral adnexectomy pelvic and inter-aortic-caval lymphadenectomy, with the excision of the multiloculated ovarian mass with haemorrhagic fluid and solid components. The histopathological exam showed an ovarian mucinous borderline tumour without stromal or regional lymph node invasion staged T1aN0M0. In the postoperative period we observed the resolution of the thrombocytopaenia, the patient's platelet count remaining stable around $120000 / \mu \mathrm{L}$.

This report highlights the causality between the complete ITP remissions after tumour excision suggesting that a possible paraneoplastic implication should always be investigated in such cases. The ovarian tumour presence may be frequently omitted and refractory thrombocytopenia attributed to other causes or classified as idiopathic.
\end{abstract}

Keywords: Thrombocytopenia; Ovarian Tumour; Surgery

\section{Abbreviations}

ITP: Immune Thrombocytopenia; CT: Computer Tomography; HE: Hematoxylin Eosin; FVIII: Factor VIII; CBC: Complete Blood Count

\section{Introduction}

Autoimmune thrombocytopenia (ITP) is an acquired immunemediated disorder characterized by early platelet destruction due to antiplatelet autoantibodies. A peripheral blood platelet count less than $100000 / \mu \mathrm{L}$ is the threshold for diagnosis [1,2]. It is estimated that approximately 100 new cases are diagnosed per $1,000,000$ persons each year, divided roughly equally between adults and children. Various solid tumours are reported to be associated with autoimmune thrombocytopenia, including breast, lung, prostate and ovarian cancers [3]. Although progress in the immunopathology of ITP has been done, pathogenesis of ITP remains yet to be determined, and currently there is no specific test that can 
diagnose this disease. It is a diagnosis of exclusion made in most cases through a careful history and physical exam, complete blood count (CBC), and review of the peripheral blood smear [4-6]. This report draws attention to a patient suffering from thrombocytopenia diagnosed with ovarian carcinoma associated with ovarian teratoma who achieved complete remission after curative surgery of both tumours.

\section{Case Report}

We describe the case of a 65-year-old woman, admitted to the surgical department with progressively worsening abdominal distension for the past 12 months. There was no history of bleeding. Her obstetrical history included 0 term births, 0 preterm births, 0 abortions, menarche at 13 and menopause beginning at age of 52 . She had no previous history of any illnesses, allergies, signs of infection or surgeries and her family history was unremarkable. At the moment of admission she wasn't under any medication.

The physical examination revealed a giant mass filling abdomen, measuring $40 \times 34 \mathrm{~cm}$, extending from pubis to epigastrium, firm to hard in consistency with restricted mobility. Auscultation of the abdomen revealed normal bowel sounds.

Complete blood count and serology revealed mild anaemia, $(\mathrm{Hb}=10 \mathrm{~g} / \mathrm{dL}$, with haematocrit 32.5\%), platelet count (PLT) of $21.000 / \mu \mathrm{L}$ and increased tumour markers CA-125 $=83.9 \mathrm{IU} / \mathrm{mL}$ $(\mathrm{N}<35 \mathrm{IU} / \mathrm{mL})$ and $\mathrm{CEA}=6.59 \mathrm{ng} / \mathrm{mL}(\mathrm{N}<5.0 \mathrm{ng} / \mathrm{mL})$. Additionally, peripheral blood showed anisocytosis with micro- and macrothrombocytes. Coagulation profile was normal. The patient was admitted with a provisional diagnosis of malignant pelvic tumour.

Ultrasound examination was suggestive of cystic mass 29/27.7/17.6 cm, mildly hypoechoic areas, internal splits and intra-tumour vascularisation.

The haematology department suspected autoimmune thrombocytopenia, and intravenous Dexamethasone was administrated with no beneficial effect on PLT or Hb levels was consulted and treatment with was recommended.

A contrast-enhanced computed tomography (CT) of abdomen + pelvis revealed a giant cystic tumour with a distinct septum and inhomogeneous internal content, which extended from the pelvis all the way through the upper abdomen, suggesting a complex left ovarian cyst. Also, the right ovary contained a haemorrhagic cyst measuring 22/16 mm. Small amount of ascites but no lymph nodes or other tumoural masses were detected.
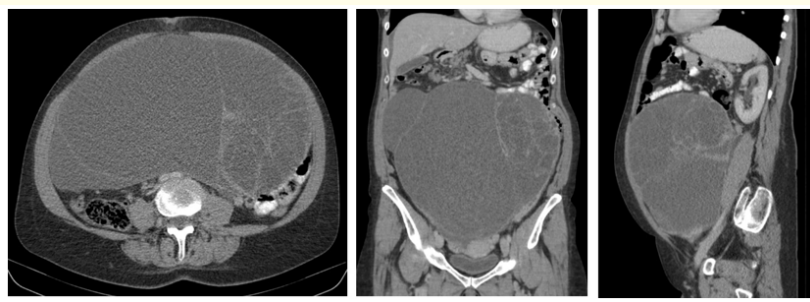

Figure 1: Preoperative CT aspect of the ovarian tumour.

Because of her increased bleeding tendency, transfusion of 6 platelet units was performed. One day after transfusion, bleeding time was greater than $20 \mathrm{~min}$ and platelet counts elevated from 16,000 to $57,000 / \mu \mathrm{L}$.

On the basis of these findings, the patient underwent surgery that revealed approximately $300 \mathrm{ml}$ of transudate-like ascitic fluid (samples were sent for bacteriological and histopathological examination); giant cystic mass originating from the left ovary with solid and semisolid areas, measuring 55/40/35 cm. Although the tumour was encapsulated, it was adherent to the posterior wall of the uterus, with adenopathy in the left parameter and pelvic region. The exploration also revealed a $3 \mathrm{~cm}$ tight ovarian cyst. The uterus was normal. No metastasis in the peritoneal cavity was observed by gross examination. Hysterectomy with bilateral adnexectomy pelvic and inter-aortic-caval lymphadenectomy was performed. There were no bleeding complications at surgery. Macroscopic examination after the operation showed a multiloculated mass with haemorrhagic fluid and solid components.

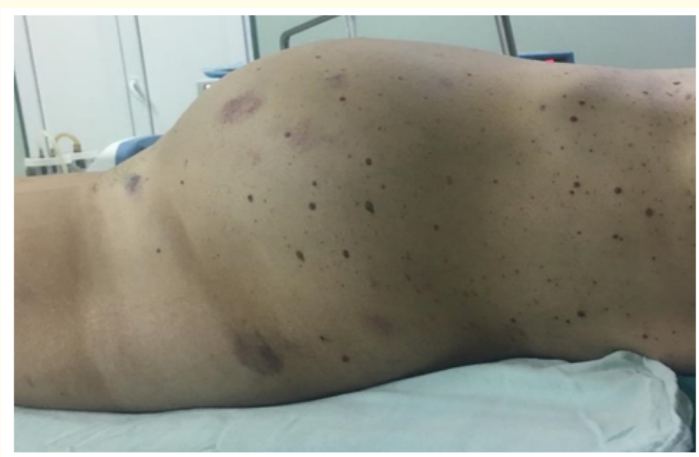

Figure 2: Preoperative aspect - visible abdominal mass. 


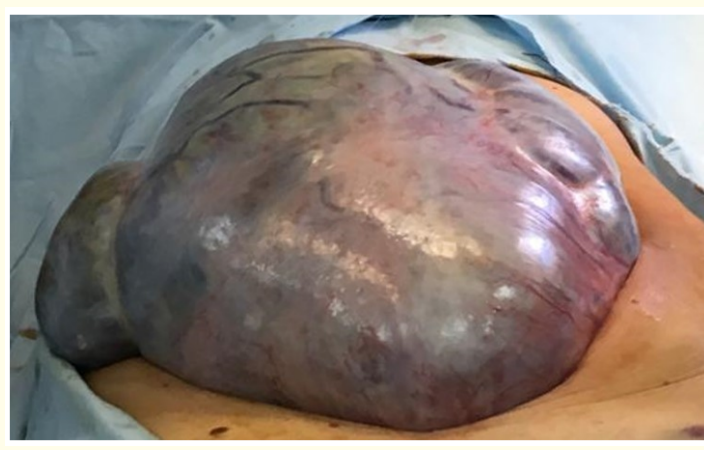

Figure 3: Intraoperative aspect of the tumour.

The histopathological exam (HP) showed an ovarian mucinous borderline tumour without stromal or regional lymph node invasion. According to the International Federation of Gynaecology and Obstetrics (FIGO) and the American Joint Committee on Cancer/ Union for International Cancer Control (AJCC/UICC) staging system, she was diagnosed as having ovarian cancer stage T1aN0M0. Although, the right ovarian cystic formation was confirmed as a mature teratoma with thyroid tissue - Struma ovarii.

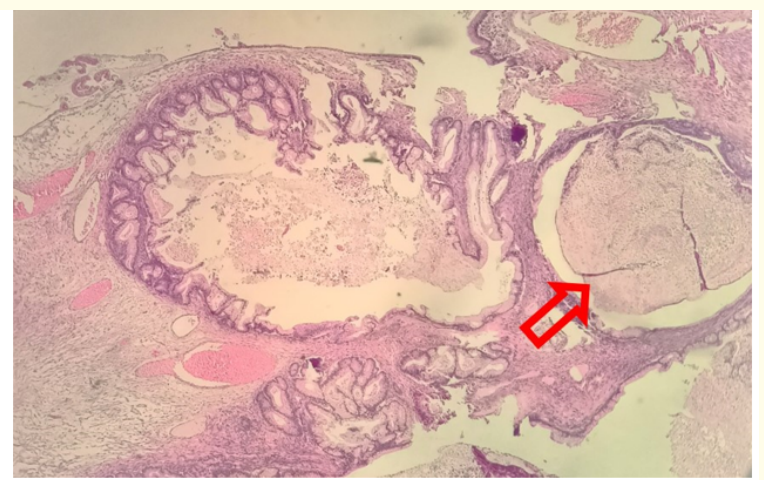

Figure 4: Mucinous ovarian tumour - HE 40x Mucinous accumulation red arrow.

On the $19^{\text {th }}$ postoperative day, physical examination revealed a mass on the left side of the abdomen, involving the left iliac fossa with bilateral lower extremity oedema. An abdominal-pelvic computed tomography (CT) scan revealed a fluid accumulation in the hypogastrium and left iliac fossa measuring about 16.5/11.5 $\mathrm{cm}$ with mass effect on the bladder, left ureter and left external iliac vasculature and another one in the right iliac fossa sized $7.6 / 3.5 / 5.6 \mathrm{~cm}$ in contact with the right pelvic ureter.

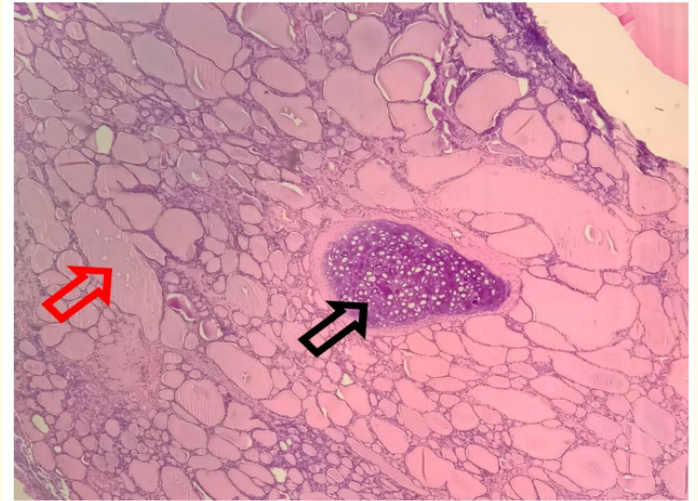

Figure 5: Ovarian teratoma showing colloidal thyroid follicles (red arrow) and cartilaginous tissue (black arrow) HE 40x.
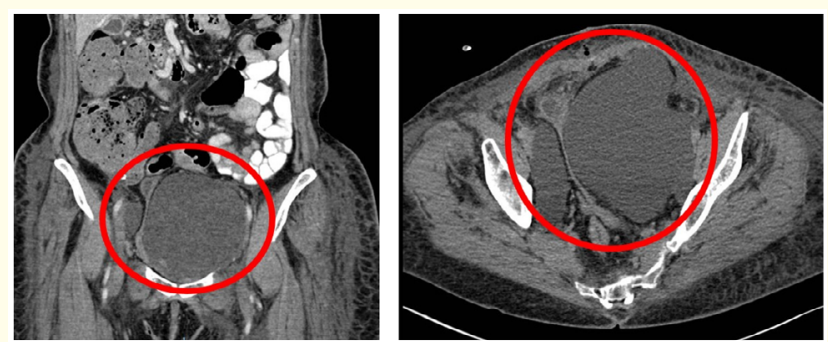

Figure 6: Postoperative CT aspect of the intraabdominal fluid accumulations - red circle.

A drain tube was introduced through the lower $1 / 3$ of the surgical wound evacuating approximately $1000 \mathrm{ml}$ serous followed a progressive decrease of the abdominal swelling in the following days. Approximately two month after discharge from hospital, platelet count remained stable around $120000 / \mu \mathrm{L}$.

Due to the slow resolution of her thrombocytopaenia with low response to transfusion therapy, the patient had to be hospitalized for 40 days. Since the peripheral blood smear only showed thrombocytes modifications only and due to the postoperative platelet count increase, the patient did further haematological investigations.

\section{Discussion}

This paper describes the unique case of a 65-year-old woman who presented with autoimmune thrombocytopenia (ITP) associated with a left giant ovarian cancer and a right mature cystic tera- 
toma and whose platelet count improved within a month after surgery. Thus, we question whether remission of the severe ITP may be induced by treatment of the primary tumors. Thrombocytopenia of autoimmune pathogenesis rarely occurs with solid tumors and has not received much attention in the oncological literature [3]. The incidence of ITP is 3 to 5 per 100,000 inhabitants is divided into primary (idiopathic) and secondary forms. Primary ITP is a diagnosis of exclusion and constitutes about $80 \%$ of diagnosed patients. In patients with solid tumors, the most common cause of thrombocytopenia is myelosuppression secondary to systemic chemotherapy or radiotherapy $[1,7]$. In this case, the patient did not receive systemic chemotherapy before the moment of diagnosis.

The occurrence of idiopathic thrombocytopenia in ovarian cancer has been reported in literature since 1991, and, more recently, a few more cases have been described and documented [8]. There is one prior case report published in 2015 that describes the case of a 49-year-old woman diagnosed with ITP in the presence of ovarian neoplasia that went in remission four days after surgery. This is the first report of a patient with ITP accompanying ovarian cancer in which the platelet count reverted to normal soon after surgery for the ovarian cancer [9]. A review published by Hudson in 1993 concluded that of all tumors, the ovarian ones seem to be one of the most immunogenic, and proposes a mechanism based on a cellmediated autoantibody production. Yet the pathogenicity of ITP in ovarian cancer remains largely unknown. Many hypothesizes have been formulated: platelet destruction or inhibition of platelet production, expression of an antigen that is immunogenic via the production of autoantibodies against platelets, involvement of antiFVIII antibodies, antiphospholipid antibodies [10,11].

The first association between ITP and solid cancer was mentioned approximately 35 years ago. Maria Theresa Krauth., et al. assessed 68 cases of this conjunction. Most often, it has been observed in association with breast and lung cancers $[3,12]$.

The ITP of severe thrombocytopenia due to an ovarian teratoma was recently analyzed in several publications. The first review published in 2009 reported a 26-year-old woman 18 weeks pregnant with the right ovarian mass [13]. The second review was published in 2018 and reported a 26-year-old female patient who was incidentally diagnosed with a $10 \mathrm{~cm}$ left ovarian mass [14]. The third case, a 41 year old patient was hospitalized for deep vein thrombo- sis in the setting of severe refractory thrombocytopenia, Abdominal CT scan showing bilateral ovarian dermoid cysts [15]. Surgical removal of the ovarian teratoma has completely resolved the severe thrombocytopenia in both patients. According to Garnick and Griffin, the tumor produces a non-immunoglobulin substance toxic to platelets or megakaryocytes [16]. Also, Khalife., et al. mentioned that the substance secreted by the teratoma may be toxic to the normal platelets with direct platelet membrane damage and that the presence of the ovarian teratoma is the specific precipitant towards the development of ITP [14]. A literature screening returned few reported cases on the co-existence of mature cystic teratoma and mucinous cystadenoma, but none in association with ITP. The pathogenic relationship between these two lesions remains unanswered [17-20].

We thus report the first such case - to our knowledge - of ovarian cancer associated with an ovarian teratoma complicated with severe and refractory ITP that was successfully cured by radical resection of both tumors. This suggests that for this patient, the ITP might have been causally related to one of the tumors, presumably through an antiplatelet antibody. We believe that removing the tumor led to remission of the ITP.

\section{Conclusion}

This report highlights the causality between the complete ITP remissions after tumour excision suggesting that a possible paraneoplastic implication should always be investigated in such cases. The ovarian tumour presence may be frequently omitted and refractory thrombocytopenia attributed to other causes or classified as idiopathic.

\section{Authors Contribution}

All authors contributed to this work equally, read and approved the final version of the paper.

\section{Conflicts of Interest}

The authors declare that there are no conflicts of interest.

\section{Bibliography}

1. Kistanguri G., et al. "Immune Thrombocytopenia". Hematology/Oncology Clinics of North America 27.3 (2013): 495-520.

2. Stasi R., et al. "Long-term outcome of otherwise healthy individuals with incidentally discovered borderline thrombocytopenia". PLOS Medicine 3 (2006): e24. 
3. Krauth MT., et al. "Paraneoplastic autoimmune thrombocytopenia in solid tumours". Clinical Reviews in Oncology/Hematology 81 (2012): 75-81.

4. Rodeghiero F., et al. "Standardization of terminology, definitions and outcome criteria in immune thrombocytopenic purpura of adults and children: report from an international working group". Blood 113 (2009): 2386-2393.

5. Abrahamson PE., et al. "The incidence of idiopathic thrombocytopenic purpura among adults: a population-based study and literature review". European Journal of Haematology 83.2 (2009): 83-89.

6. Chehal A., et al. "Idiopathic thrombocytopenic purpura and ovarian cancer". European Journal of Gynaecological Oncology 24 (2003): 539-540.

7. Lambert MP., et al. "Clinical updates in adult immune thrombocytopenia”. Blood 129.21 (2017): 2829.

8. Bellucci S., et al. "Association of autoimmune thrombocytopenic purpura (AITP), Graves' disease and ovarian carcinoma". Nouvelle Revue Francaise d'Hematologie 33 (1991): 307-309.

9. Shimada T., et al. "Immune thrombocytopenia associated with solid cancer". Journal of Obstetrics and Gynaecology Research 41.9 (2015): 1495-1498.

10. Hudson CN., et al. "Paraneoplastic syndromes in patients with ovarian neoplasia". Journal of the Royal Society of Medicine 86 (1993): 202-204.

11. Schwartz KA., et al. "Immune-mediated platelet destruction and thrombocytopenia in patients with solid tumours". The British Journal of Haematology 51 (1982): 17-24.

12. Kim HD., et al. "A syndrome resembling idiopathic thrombocytopenic purpura in 10 patients with diverse forms of cancer". The American Journal of Medicine 67 (1979): 371-377.

13. Soma-Pillay P., et al. "A rare case of idiopathic thrombocytopenia in association with an ovarian teratoma in pregnancy". Obstetric Medicine 2.3 (2009): 126-127.

14. Khalife D., et al. "Ovarian teratoma: yet another cause of immune thrombocytopenia". The International Journal of Women's Health 10 (2018): 567-570.

15. Ai S S Y., et al. "Immune thrombocytopenia in the setting of ovarian dermoid cysts: implications for diagnosis and management" The Internal Medicine Journal 48.10 (2018): 12751276.
16. Garnick MB., et al. "Idiopathic thrombocytopenia in association with extragonadal germ cell cancer". Annals of Internal Medicine 98.6 (1983): 926-927.

17. Tang P., et al. "Mature cystic teratoma of the ovary associated with complete colonic wall and mucinous cystadenoma". Annals of Clinical and Laboratory Science 33.4 (2003): 465-470.

18. Shen Q., et al. "Co-existing mature cystic teratoma and borderline ovarian mucinous cystadenoma: a report of three cases". International Journal of Clinical and Experimental Medicine 11.5 (2018): 5227-5231.

19. Roy S., et al. "Mature Cystic Teratoma with Co-existent Mucinous Cystadenocarcinoma in the same Ovary-A Diagnostic Dilemma". Journal of Clinical and Diagnostic Research 10.12 (2016): ED11-ED13.

20. Ueda G., et al. "Adenocarcinoma in a benign cystic teratoma of the ovary: Report of a case with a long survival period". Gynecologic Oncology (1993): 259-263.

\section{Volume 5 Issue 12 December 2021 (C) All rights are reserved by Ioan Corneliu Tanase., et al.}

